\section{Schaderpreis geht an Meinhard Miegel}

Prof. Dr. Meinhard Miegel erhält den diesjährigen Schaderpreis für seine herausragende Rolle im Dialog zwischen Gesellschaftswissenschaften und der politischen Praxis. Meinhard Miegel ist geschäftsführender Vorstand und wissenschaftlicher Leiter des Bonner Instituts für Wirtschaft und Gesellschaft.

Der Schaderpreis wird Meinhard Miegel in Anerkennung seiner langjährigen wissenschaftlichen Arbeit zur Analyse und Bestimmung gesellschaftlicher Zukunftsprobleme zugesprochen. Miegel hat mit seinen Arbeiten frühzeitig und wiederholt auf die Folgen des demographischen und sozialen Wandels für die angestammten Einrichtungen des Wohlfahrtsstaates aufmerksam gemacht und Lösungsstrategien entworfen.

Heute werden viele der Probleme der Alterssicherungssysteme, der Arbeitsmärkte der $\mathrm{Zu}-$ kunft und des Wohnens politisch diskutiert, für die Miegel vor Jahr und Tag fundierte Analysen vorgelegt und zukunftsträchtige Entscheidungen angemahnt hat. Die Schader-Stiftung würdigt mit ihrer Auszeichnung einen hervorragenden Wis- senschaftler, der sich in vielfältigen Formen direkt und über die Medien in den gesellschaftspolitischen Willensbildungsprozess eingemischt und dabei durch seine Arbeit und sein Engagement beispielhaft die Praxisrelevanz von Gesellschaftstheorie und Grundlagenforschung demonstriert hat.

Die Preisverleihung findet Ende des Jahres in Darmstadt statt. Mit dem Schaderpreis zeichnet die Schader-Stiftung Gesellschaftswissenschaftler aus, die aufgrund ihrer wegweisenden wissenschaftlichen Arbeit und durch ihr vorbildliches Engagement mit der Praxis einen Beitrag zur Lösung gesellschaftlicher Aufgaben geleistet haben. Der Preis in Höhe von DM 30.000 wird seit 1993 jährlich verlichen. Das Kuratorium der Stiftung ist die Preisjury.

Die Schader-Stiftung ist eine gemeinnützige rechtsfähige Stiftung bürgerlichen Rechts mit Sitz in Darmstadt. Sie hat es sich zum Ziel gesetzt, durch die Förderung von Kommunikation und Kooperation zwischen Gesellschaftswissenschaften und Praxis einen Beitrag zur Weiterentwicklung des Gemeinwesens zu leisten.

Schader-Stiftung, Darmstadt 